\title{
Deplete and Delirious: A Report of Two Cases
}

\author{
Amanda Bryson ${ }^{\mathrm{a}}$, Melissa Yacur ${ }^{\mathrm{b}, \mathrm{d}}$, Meeta Desaic, \\ Eileen Hennrikus ${ }^{b}$
}

\begin{abstract}
A diagnosis of thiamine deficiency and Wernicke's encephalopathy should be considered in all elderly patients presenting to the emergency department with acute neurological changes. Physicians have an increased suspicion for the diagnosis in alcoholic patients, but unfortunately this condition is often missed in non-alcoholic patients. Timely diagnosis and treatment of these conditions is imperative due to the associated morbidity and mortality. In this report, we describe two cases of elderly patients who experience severe neurological disturbances secondary to Wernicke's encephalopathy. Both patients' conditions were worsened with an infusion of dextrose containing fluids and promptly improved with infusion of thiamine. We believe that acutely ill, elderly patients are particularly susceptible to thiamine deficiency and the development of Wernicke's encephalopathy. The recognition of this population as especially vulnerable is critical in order to improve long-term neurological outcomes in these patients.
\end{abstract}

Keywords: Thiamine; Wernicke's encephalopathy; Elderly

\section{Introduction}

Wernicke's encephalopathy (WE) is a neuropsychiatric emergency caused by thiamine deficiency and is characterized by ocular disturbances, gait ataxia, and confusion [1]. Physicians readily suspect and often prophylactically treat thiamine deficiency in alcoholic patients to prevent the serious consequences of irreversible encephalopathy, coma, and death $[1,2]$.

Additional risk factors for thiamine deficiency have been discovered, but remain less recognized in clinical practice compared to alcoholism. Gastrointestinal surgeries or frequent

\footnotetext{
Manuscript accepted for publication March 08, 2017

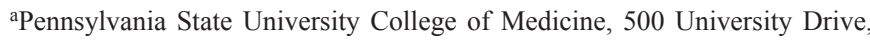
Hershey, PA, USA

bepartment of Internal Medicine, Pennsylvania State University College of Medicine, 500 University Drive, Hershey, PA, USA

${ }^{\mathrm{c} V}$ irginia Tech Carilion School of Medicine, Roanoke, VA, USA

${ }^{\mathrm{d} C}$ Corresponding Author: Melissa Yacur, Department of Internal Medicine, Pennsylvania State University College of Medicine, 500 University Drive, Hershey, PA, USA. Email: myacur@pennstatehealth.psu.edu
}

doi: https://doi.org/10.14740/jmc2802e vomiting or diarrhea contribute to thiamine deficiency as a result of poor digestion, malabsorption, and decreased oral intake [3]. Infection and cancer result in hypermetabolic states, thus diminishing the body's available thiamine $[1,3,4]$. Despite the discovery of other risk factors, the clinical suspicion for the diagnosis of WE in non-alcoholic patients remains low [2]. Based on clinical observation, elderly patients may be at increased risk for thiamine deficiency and the development of WE. We report two cases of acutely ill, non-alcoholic, elderly patients developing WE with remarkable recoveries secondary to thiamine replacement.

\section{Case Reports}

\section{Case 1}

An 89-year-old man with parkinsonism, who lived at home alone with no significant alcohol use, presented to the emergency department (ED) with acute onset of generalized weakness. He required ICU admission for severe sepsis, with a leukocytosis $(15,000$ cells $/ \mu \mathrm{L})$ and lactic acidosis $(2.5 \mathrm{mmol} / \mathrm{L})$. Within $24 \mathrm{~h}$ of antibiotic treatment and normal saline resuscitation, he significantly improved and was pleasant, cooperative, alert, and oriented. While NPO for a speech evaluation for dysphagia, he became hypoglycemic $(45 \mathrm{mg} / \mathrm{dL})$ and was started on a continuous infusion of dextrose containing fluids. The following morning, he was acutely delirious. His confusion and agitation worsened until he became unresponsive. A workup revealed a prealbumin of $6 \mathrm{mg} / \mathrm{dL}$ and a lactate of $4.5 \mathrm{mmol} / \mathrm{L}$. There was no evidence of recurrent sepsis or acute intracranial abnormality. His only new focal neurological finding was ophthalmoplegia. A thiamine level was drawn and he was empirically started on thiamine $500 \mathrm{mg}$ intravenously (IV) every 8 $\mathrm{h}$ and IV magnesium, a cofactor for thiamine. Over the next $24 \mathrm{~h}$, he became responsive and continued to improve back to his baseline mental status. Thiamine level later returned as $<7$ $\mathrm{nmol} / \mathrm{L}$ (normal 8 - $30 \mathrm{nmol} / \mathrm{L}$ ). He was ultimately discharged home on oral thiamine $100 \mathrm{mg}$ daily.

\section{Case 2}

A 64-year-old female with a past medical history of diabetes mellitus, non-alcoholic fatty liver disease, multiple transient ischemic attacks, hypothyroidism, and ulcerative colitis pre- 


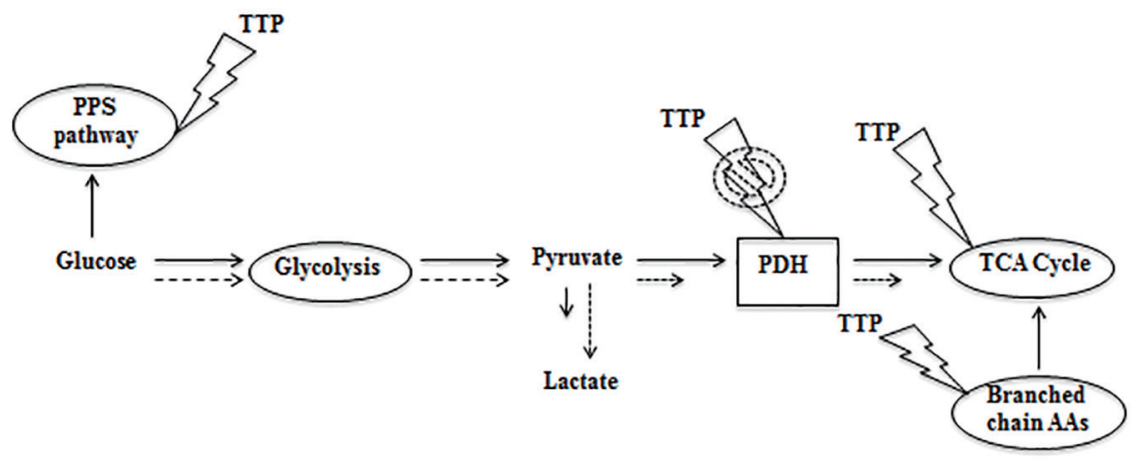

Figure 1. In absence of thiamine, glucose metabolism is shunted towards the production of lactate (dotted lines). PPS: pentose phosphate shunt; PDH: pyruvate dehydrogenase; TCA: tricarboxylic acid; AA: amino acids; TTP: thiamine pyrophosphate.

sented to the ED due to worsening acute on chronic encephalopathy. Her baseline mental status had been declining over the past 2 years and recently required assistance with her activities of daily living as well as increased memory loss associated with fluctuating episodes of confusion. On the day of admission, she had become increasingly agitated, confused, and unable to ambulate. Upon evaluation in the ED, she was afebrile, but had sinus tachycardia and tachypnea. She was unable to follow commands or respond to questions. She was very agitated and continually repeated "help me" and "how many". Her eyelids were tightly closed, but when manually lifted her eyes roamed horizontally. Her extremities were stiff with $3+$ deep tendon reflexes. A workup revealed a lactic acid of 5.7 $\mathrm{mmol} / \mathrm{L}$ without evidence of infection and an unremarkable head CT. She was started on dextrose containing fluids and broad-spectrum antibiotics empirically. Following this, her mental status worsened, her agitation increased, and she began thrashing around. A thiamine level was drawn and she was started on IV thiamine. Within $72 \mathrm{~h}$, she was sitting up, eating breakfast, and conversing with her husband. She steadily improved, surpassing her previous baseline and was discharged home on oral thiamine $100 \mathrm{mg}$ daily. Her thiamine level later returned below normal range.

\section{Discussion}

Thiamine is a water-soluble vitamin that serves as a cofactor for essential metabolic reactions [5]. Thiamine is readily available in certain meats, whole grains, legumes, and thiamineenriched foods [6]. It is recommended that healthy adults consume $1.4 \mathrm{mg}$ daily [3].

In healthy individuals, thiamine stores can be depleted in 18 days [3]. When thiamine-deficient patients receive dextrose, glucose cannot be aerobically metabolized through the Krebs cycle, but rather, it is shunted towards anaerobic metabolism and lactate production (Fig. 1). As thiamine levels decrease, thiamine-dependent processes decline, including metabolism of carbohydrates, branched chain amino acids, maintenance of neural myelin sheaths, and synthesis of neurotransmitters [6]. The resultant neurotoxic edema and oxidative stress form the cortical lesions found in $\mathrm{WE}[1,3,6]$.
We propose that acutely ill, elderly patients are vulnerable to thiamine deficiency and the subsequent development of WE. When well, these patients may have borderline levels of thiamine due to poor nutrition. When ill, these patients are at risk for thiamine deficiency due to a hypermetabolic state that rapidly consumes thiamine [4]. Additionally, nutritional intake is frequently limited during acute hospitalization and can exacerbate thiamine deficiency as seen in our patient discussed in case 1 . The cases reported demonstrate that the combination of these factors makes elderly patients particularly susceptible to thiamine deficiency and its sequela.

Thiamine deficiency and progression to $\mathrm{WE}$ is preventable and treatable [2]. Prompt initiation of IV thiamine is low-risk and is paramount for achieving favorable outcomes in these patients [1]. Physicians should consider thiamine supplementation in elderly, non-alcoholic patients with marginal nutrition who present acutely ill.

\section{Funding}

None.

\section{Conflicts of Interest}

None of the authors have any conflicts of interest

\section{Verification}

All authors had access to data and a role in writing the manuscript.

\section{References}

1. Flynn A, Macaluso M, D'Empaire I, Troutman MM. Wernicke's Encephalopathy: Increasing Clinician Awareness of This Serious, Enigmatic, Yet Treatable Disease. Prim Care Companion CNS Disord. 2015;17(3).

2. Scalzo SJ, Bowden SC, Ambrose ML, Whelan G, Cook 
MJ. Wernicke-Korsakoff syndrome not related to alcohol use: a systematic review. J Neurol Neurosurg Psychiatry. 2015;86(12):1362-1368.

3. Sechi G, Serra A. Wernicke's encephalopathy: new clinical settings and recent advances in diagnosis and management. Lancet Neurol. 2007;6(5):442-455.

4. Isenberg-Grzeda E, Alici Y, Hatzoglou V, Nelson C, Breitbart W. Nonalcoholic Thiamine-Related Encephalopathy (Wernicke-Korsakoff Syndrome) Among Inpatients
With Cancer: A Series of 18 Cases. Psychosomatics. 2016;57(1):71-81.

5. Andersen LW, Mackenhauer J, Roberts JC, Berg KM, Cocchi MN, Donnino MW. Etiology and therapeutic approach to elevated lactate levels. Mayo Clin Proc. 2013;88(10):1127-1140.

6. Hutcheon DA. Malnutrition-induced Wernicke's encephalopathy following a water-only fasting diet. Nutr Clin Pract. 2015;30(1):92-99. 